\title{
Left Ventricular Assist Devices and Small Body Surface Area

\author{
- A Clinical Concern? -
}

\author{
Michael Ibrahim, MD, PhD; Arman Kilic, MD; Pavan Atluri, MD
}

$\mathbf{L}$ eft ventricular assist devices (LVADs) are sophisticated multi-component devices (Figure). ${ }^{1}$ Their proper functioning and optimal biomechanics depend on appropriate interaction between all the component parts and the heart, aorta, chest cavity and in some devices the abdominal compartment. ${ }^{2}$ Early LVADs were large, pulsatile devices that were not suitable for smaller patients; but newer generation continuous-flow LVADs are smaller, allowing for implantation in patients with a lower body surface area (BSA). ${ }^{3}$ The average BSA of patients receiving pulsatile vs. latest generation continuous-flow devices has accordingly fallen. ${ }^{4}$ The importance of matching the device to the body habitus is illustrated by cases in which post-implantation weight loss can result in changes to the physical interaction between the device and heart, resulting in either poor LVAD performance or even lethal arrhythmias related to cannula to ventricular cavity geometric changes. Therefore, both for appropriate hemodynamic support and for correct anatomical placement, proper size matching for the device is an important consideration both at the time of implantation and in the postoperative period. Patients with BSA $<1.5 \mathrm{~m}^{2}$ represent an especially challenging group.

\section{Article p 1931}

Heart failure patients, who often have a high burden of obesity, hyperlipidemia, and the metabolic syndrome, typically have a BSA of approximately $2.0 \mathrm{~m}^{2} .{ }^{5-7}$ The design and biomechanics of LVADs are typically geared toward this target BSA. Indeed, many LVADs have a lower limit of BSA in which they can be implanted: this can range from $1.1 \mathrm{~m}^{2}$ for the DuraHeart LVAD, ${ }^{7}$ to a minimum of $1.7 \mathrm{~m}^{2}$ for the CardioWest total artificial heart. ${ }^{8}$ The HeartMate II LVAD is generally thought to have a lower limit of a BSA of $1.4 \mathrm{~m}^{2}$, though isolated reports have described implantation in patients with smaller BSA. ${ }^{6}$

Implantation in smaller patients with a BSA $<1.5 \mathrm{~m}^{2}$ is potentially problematic for several theoretical reasons. Given the same device size, implantation into small patients necessitates lower pump speeds to meet the circulatory demands, and potentially increases the contact time of blood on the pump surface, theoretically increasing the potential for clot formation. In addition, the anatomic factors just described may play a role, in having to arrange all the LVAD elements into a smaller space without tension, torsion or kinking while ensuring optimal alignment.

In this issue of the Journal, Ono et a ${ }^{9}$ investigate the role of

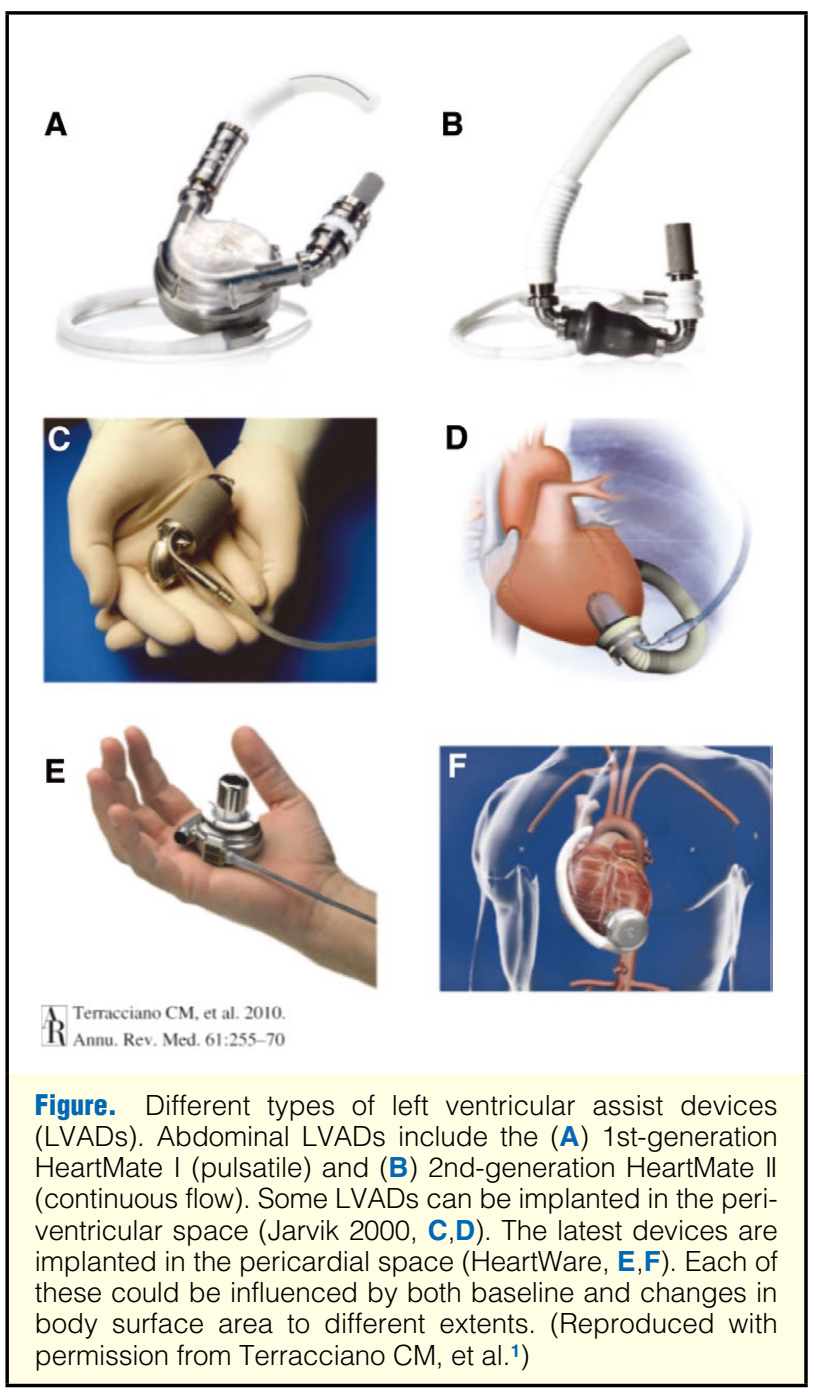

The opinions expressed in this article are not necessarily those of the editors or of the Japanese Circulation Society.

Received July 26, 2016; accepted July 27, 2016; released online August 5, 2016

Division of Cardiovascular Surgery, Hospital of the University of Pennsylvania, Philadelphia, PA, USA

Mailing address: Pavan Atluri, MD, Division of Cardiovascular Surgery, Hospital of the University of Pennsylvania, 3400 Spruce Street,

6 Silverstein Pavilion, Philadelphia, PA 19104, USA. E-mail: Pavan.Atluri@uphs.upenn.edu

ISSN-1346-9843 doi:10.1253/circj.CJ-16-0749

All rights are reserved to the Japanese Circulation Society. For permissions, please e-mail: cj@j-circ.or.jp 
BSA on clinical outcomes for the HeartMate II LVAD (used as a bridge to transplant) by retrospectively comparing clinical outcomes of death, New York Heart Association functional class and common adverse events in patients with a BSA of less than vs. greater than $1.5 \mathrm{~m}^{2}$ in a multicenter Japanese population. There were more than double the number of patients in the BSA $>1.5 \mathrm{~m}^{2}$ group, who were also significantly more likely to have ischemic cardiomyopathy. The 2 groups also differed in that the smaller cohort were more likely to be young, female and to have had a prior LVAD. They were otherwise well matched.

The 2 groups were equivalent in mortality rate at 1 year and in improvement in NYHA functional class. There was no difference in the overall rate of adverse events, with more common driveline infections in the smaller BSA group but more common embolic stroke in the larger BSA group. Hemorrhagic stroke rates were equivalent between the 2 groups.

Previous studies based on the INCOR LVAD have indicated that patients with a small BSA are at increased risk of death from stroke or bleeding. ${ }^{10,11}$ This has been ascribed to the lower flow rates in smaller patients and potential anatomical considerations. Although in the present study pump speed tended to be slower in the smaller BSA cohort of patients, the difference did not achieve statistical significance. Additionally, there was no statistically significant difference in the INR of both groups. As the authors suggest, this indicates that when the INR is tightly controlled, lower pump speeds can be mitigated. Overall pump speeds were generally low in both groups, at $8,657 \pm 4,238 \mathrm{rpm}$ even in the BSA $>1.5 \mathrm{~m}^{2}$ group. This could be related to the fact that the average BSA in the larger group of patients was $1.69 \pm 0.78$, which is smaller than the average BSA of heart failure patients in the Western world who typically have BSA close to 2.0.1,5

It is notable that the overall rates of death and adverse events reported in this study are low, and the rate of gastrointestinal bleeding is especially so. Therefore, even the "larger" BSA group demonstrated satisfactory clinical outcomes in a group of patients with a BSA that is smaller than their Western counterparts. However, this does raise a potential concern over the lack of a control group of "typical" patients, with a BSA closer to the target BSA of the HeartMate II of $2.0 \mathrm{~m}^{2}$. For example, in a study of the INCOR LVAD ${ }^{10}$ significantly higher rates of death from stroke or systemic bleeding were found using a BSA cut off of $1.867 \mathrm{~m}^{2}$. This is a larger minimum cutoff BSA than the average BSA in the "large" group of patients in the present study. It could be interesting to pool data from several national registries to compare matched patients with a wider range of BSA.
The Japanese experience taken as a whole, and especially in the BSA $<1.5 \mathrm{~m}^{2}$ group, shows that with careful attention to implantation technique, and especially cannula positioning and pocket construction, and meticulous postoperative anticoagulation protocols, excellent outcomes can be achieved in small recipients of LVADs, running at the lower end of pump speed. These data are a testament to the technological advancements that have enabled a rapid reduction in the size and thrombogenicity of LVADs over the past several decades, as well as optimal anticoagulation management. The latest generation of intrapericardial LVADs, including the HeartWare and HeartMate III, may be used to support adult patients with BSA $>1.2 \mathrm{~m}^{2}$. In the decades to come, we can expect further advancements in safety and further reductions in size, enabling less troublesome implantation in even the smallest patients.

\section{References}

1. Terracciano CM, Miller LW, Yacoub MH. Contemporary use of ventricular assist devices. Апnи Rev Med 2010; 61: 255-270.

2. Imamura T, Kinugawa K, Kato N, Muraoka H, Fujino T, Inaba T, et al. Late-onset right ventricular failure in patients with preoperative small left ventricle after implantation of continuous flow left ventricular assist device. Circ J 2014; 78: 625-633.

3. Gafoor S, Franke J, Lam S, Reinartz M, Bertog S, Vaskelyte L, et al. Devices in heart failure: The new revolution. Circ J 2015; 79: 237 244.

4. Kirklin JK, Naftel DC, Pagani FD, Kormos RL, Stevenson L, Miller $\mathrm{M}$, et al. Long-term mechanical circulatory support (destination therapy): On track to compete with heart transplantation? J Thorac Cardiovasc Surg 2012; 144: 584-603; discussion 597-598.

5. Goldstein DJ. Worldwide experience with the MicroMed DeBakey Ventricular Assist Device as a bridge to transplantation. Circulation 2003; 108(Suppl 1): II272-II277.

6. Miller LW, Pagani FD, Russell SD, John R, Boyle AJ, Aaronson $\mathrm{KD}$, et al. Use of a continuous-flow device in patients awaiting heart transplantation. N Engl J Med 2007; 357: 885-896.

7. Morshuis M, El-Banayosy A, Arusoglu L, Koerfer R, Hetzer R, Wieselthaler G, et al. European experience of DuraHeart magnetically levitated centrifugal left ventricular assist system. Eur J Cardiothorac Surg 2009; 35: 1020-1027; discussion 1027-1028.

8. Platis A, Larson DF. CardioWest temporary total artificial heart. Perfusion 2009; 24: 341-346.

9. Ono M, Sawa Y, Nakatani T, Tominaga R, Matsui Y, Yamazaki K, et al; on behalf of Japanese HeartMate II investigators. Japanese multicenter outcomes with the HeartMate II left ventricular assist device in patients with small body surface area. Circ J 2016; 80: $1931-1936$

10. Komoda T, Drews T, Hetzer R, Lehmkuhl HB. Lower body surface area is highly related to mortality due to stroke or systemic bleeding in patients receiving an axial flow blood pump as a left ventricular assist device. Eur J Cardiothorac Surg 2013; 43: 1036-1042.

11. Komoda T, Drews T, Hetzer R, Lehmkuhl HB. Adult candidates for heart transplantation with larger body surface area have better prognosis on waiting list after progression to critically ill status. Eur $J$ Cardiothorac Surg 2011; 39: 317-322. 